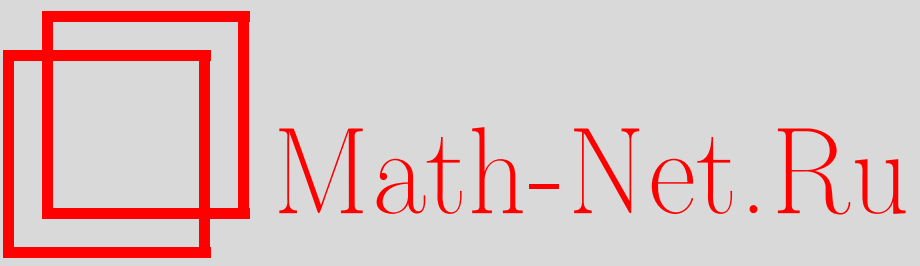

М. Фарбер, Сигнатура в терминах чисел Новикова, УМН, 1999, том 54, выпуск 1, 209-212

DOI: https://doi.org/10.4213/rm121

Использование Общероссийского математического портала Math-Net.Ru подразумевает, что вы прочитали и согласны с пользовательским соглашением

http://www.mathnet.ru/rus/agreement

Параметры загрузки:

IP : 18.234 .156 .22

26 апреля 2023 г., 06:10:36 


\title{
СИГНАТУРА В ТЕРМИНАХ ЧИСЕЛ НОВИКОВА
}

\author{
M. ФАРБЕР
}

1. ТЕОРемА. Пусть $M^{2 n}$ - симплектическое многообразие с симплектическим действием окружности, которое имеет лишь изолированные неподвижные точки. Тогда сигнатура $M$ задается следующей формулой:

$$
\sigma(M)=b_{0}(\xi)-b_{2}(\xi)+b_{4}(\xi)-b_{6}(\xi)+\cdots+(-1)^{n} b_{2 n}(\xi)
$$

где $\xi \in H^{1}(M ; \mathbb{R})$ обозначает класс когомологий обобщенного отображсния моментов, а $b_{i}(\xi)$ - соответствующее число Новикова.

Это обобшает теорему, доказанную в [1], в которой речь шла о гамильтоновых действиях окружности. В гамильтоновом случае $\xi=0$, а соответствующие числа Новикова становятся обычными числами Бетти $b_{i}(\xi)=b_{i}(M)$.

2. Поясним используемые в формулировке теоремы 1 понятия.

Вначале заметим, что любое симплектическое многообразие обладает канонической ориентацией, и сигнатура $\sigma(M)$ рассматривается по отношению к этой ориентации.

Пусть $\omega$ обозначает симплектическую форму на $M$. Действие окружности $S^{1}$ предполагается симплектическим, что означает, что для любого $g \in S^{1}$ имеет место $g^{*} \omega=\omega$. Пусть $X$ - векторное поле, порождаюшее $S^{1}$-действие. Тогда

$$
\theta=\iota(X) \omega
$$

- замкнутая 1-форма на $M$, называемая обобщенным отображением моментов. Мы рассматриваем класс когомологий де Рама $\xi=[\theta] \in H^{1}(M ; \mathbb{R})$ формы $\theta$.

Определение чисел Новикова $b_{i}(\xi)$ можно найти в [2]-[4].

При нечетном $n$ обе части формулы (1) обращаются в нуль. Здесь мы используем соглашение о том, что сигнатура любого $4 k+2$-мерного многообразия равна нулю. Правая часть (1) обрашается в нуль благодаря соотношению $b_{i}(\xi)=b_{2 n-i}(\xi)$, которое вытекает непосредственно из определения чисел Новикова (см. [2; определение 1.2]) и классической двойственности Пуанкаре.

\footnotetext{
Частично поддержано EPSRC Visiting Fellowship.
} 
3. Доказательство теоремы 1. Первая часть доказательства вполне аналогична рассуждениям из [1]. Вторая часть доказательства использует неравенства Новикова-Морса, установленные в [2].

Для любой неподвижной точки $p \in M$ действия окружности мы имеем линейное представление окружности $S^{1}$ на касательном пространстве $T_{p}(M)$, которое не имеет неподвижных векторов. Это определяет комплексную структуру на $T_{p}(M)$, а значит и каноническую ориентацию. Мы положим $\eta(p)=+1$ или $\eta(p)=-1$ в зависимости от того, совпадает или нет эта ориентация с ориентацией $T_{p}(M)$, задаваемой симплектической формой.

ПримеР. Для стандартного врашения двумерной сферы $S^{2}$ вокруг северного и южного полюсов $p_{N}$ и $p_{S}$, мы имеем $\eta\left(p_{N}\right)=1$ и $\eta\left(p_{S}\right)=-1$.

B [1] при помощи теоремы Атьи-Ботта о неподвижных точках было доказано, что

$$
\sigma(M)=\sum \eta(p)
$$

где сумма берется по всем неподвижным точкам $p$ действия окружности.

Применим теорему 0.3 из [2] к обобшенному отображению моментов $\theta$, задаваемому (2). Критическими точками $\theta$ являются в точности неподвижные точки $p$ действия окружности. Хорошо известно (см. [5; $§ ~ 2.1,2.2]$, а также [1]), что все эти критические точки являются невырожденными. Кроме того, индексы всех критических точек $\theta$ являются четными и

$$
\eta(p)=(-1)^{\operatorname{ind}(p) / 2}
$$

см. [1], [5]. Применяя теорему 0.3 из [2], мы получаем, что многочлен Mopca (Morse counting polynomial)

$$
\mathscr{M}_{\theta}(\lambda)=\sum c_{j}(\theta) \lambda^{j}
$$

содержит только члены четной степени (где $c_{j}(\theta)$ обозначает число критических точек $\theta$, имеющих индекс $j)$. Таким образом, многочлен $\mathscr{Q}(\lambda)$ в формуле $(0.7)$ из [2] обрашается в нуль (в силу принципа лакун Mopca (Morse lacunary principle)). Следовательно, мы получаем, что в нечетных размерностях все числа Новикова $b_{2 j-1}(\xi)$ обрашаются в нуль, а для чисел Новикова в четных размерностях мы имеем

$$
b_{2 j}(\xi)=c_{2 j}(\theta) \text {. }
$$

Из (3) и (4) мы находим $\sigma(M)=\sum(-1)^{j} c_{2 j}(\theta)$. Сопостовляя это с формулой (5), мы завершаем доказательство.

Ниже мы рассмотрим еще одно обобщение теоремы из [1]: мы будем допускать симплектические действия окружности с множеством неподвижных точек более общего вида.

Ориентированное многообразие $N$ назьвается $i$-многообразием (где $i=\sqrt{-1}$ ), если все его нечетномерные числа Бетти $b_{2 j-1}(N)$ обрашаются в нуль, а сигнатура $\sigma(N)$ равна $\sum_{j=0}^{\operatorname{dim}(N) / 2}(-1)^{j} b_{2 j}(N)$. Другими словами, сигнатура $N$ получается вычислением многочлена Пуанкаре $N$ в точке $\lambda=i$. 
Точка (вместе с ее канонической ориентацией) является $i$-многообразием. Любое комплексное проективное пространство $\mathbb{C P}^{n}$ (с канонической ориентацией) также является $i$-многообразием. Если $N_{1}$ и $N_{2}$ являются $i$-многообразиями, то их произведение $N_{1} \times N_{2}$ также является $i$-многообразием.

Результат [1] можно переформулировать следуюшим образом: любое симплектическое многообразие, допускающее гамильтоново действие окружности с изолированными неподвижными точками является $i$-многообразием. Это дает множество примеров $i$-многообразий.

Дополнительные примеры $i$-многообразий можно найти в работе Д. Мецлера (D. Metzler) [6].

Теорема 1 остается верной в случае, когда связными компонентами множества неподвижных точек действия окружности являются $i$-многообразия (вместо изолированных точек):

4. Теорема. Пусть $M^{2 n}$ - симплектическое многобразие с симплектическим действием окружности. Предположим, что каждая компонента множества неподвижных точек является $i$-многообразием. Тогда сигнатура $M$ может быть выражена в терминах чисел Новикова следующим образом

$$
\sigma(M)=\sum_{j=0}^{n}(-1)^{j} b_{2 j}(\xi),
$$

где $\xi \in H^{1}(M ; \mathbb{R})$ - класс когомологий обобщенного отображения моментов.

Заметим, что для каждой связной компоненты $Z \subset M$ множества неподвижных точек действия окружности, $\left.\omega\right|_{Z}$ является симплектической формой на $Z$, см. [5], где $\omega$ обозначает симплектическую форму на $M$. Таким образом, $Z$ обладает канонической ориентацией, и в теореме 5 мы предполагаем, что $Z$ является $i$-многообразием по отношению к этой ориентации.

5. Доказательство теоремы 4. Для каждой компоненты связности $Z \subset M$ множества неподвижных точек определим знак $\eta(Z)= \pm 1$ следующим образом. Ориентация касательного расслоения к $M$ и ориентация касательного расслоения к $Z$ (определяемая симплектической формой) определяют ориентацию нормального расслоения $\nu(Z)$ к $Z$ в $M$. Другая ориентация $\nu(Z)$ определяется действием окружности: для любой точки $p \in Z$ окружность $S^{1}$ действует на $\nu_{p}(Z)$ без неподвижных векторов и, следовательно, определяет комплексную структуру и ориентацию пространства $\nu_{p}(Z)$. Мы положим $\eta(Z)=1$, если эти две ориентации пространства $\nu_{p}(Z)$ согласованы, и $\eta(Z)=-1$, если эти две ориентации $\nu_{p}(Z)$ противоположны.

Используя рассуждения, аналогичные рассуждениям из [1], мы получаем следуюшую формулу для сигнатуры (обобшаюшую формулу (3))

$$
\sigma(M)=\sum_{Z} \eta(Z) \sigma(Z),
$$

где сумма берется по множеству связных компонент $Z$ множества неподвижных точек. Используя, как и вьше, теорему 0.3 из [2] и принцип лакун, мы находим

$$
\sum_{Z} \lambda^{\operatorname{ind}(Z)} \mathscr{P}_{Z}(\lambda)=\sum_{j \geqslant 0} \lambda^{j} b_{j}(\xi),
$$


где сумма в левой части берется по всем связным компонентам $Z$ множества неподвижных точек, а $\mathscr{P}_{Z}(\lambda)$ обозначает многочлен Пуанкаре $Z$. Отсюда мы получаем (используя наше предположение о том, что $\mathscr{P}_{Z}(\lambda)$ не содержит нечетных степеней), что все нечетномерные числа Новикова $b_{2 j-1}(\xi)$ обращаются в нуль.

Аналогично (4), мы имеем

$$
\eta(Z)=(-1)^{\operatorname{ind}(Z) / 2}
$$

Отсюда, подставляя $\lambda=i$ в (8) и используя наше предположение $\sigma(Z)=\mathscr{P}_{Z}(i)$, мы получаем (6).

6. СлЕДСтвИЕ. Пусть $M^{2 n}$ - симплектическое многообразие с гамильтоновым действием окружности, для которого кажсдая компонента множества неподвижных точек является $i$-многообразием. Тогда $M$ является $i$-многообразием.

\section{СПИСОК ЛИТЕРАТУРЫ}

[1] Jones J. D.S., Rawnsley J.H. Hamiltonian circle actions on symplectic manifolds and the signature // J. Geom. Phys. 1997. V. 23. P. 301-307.

[2] Braverman M., Farber M. Novikov type inequalities for differential forms with nonisolated zeros // Math. Proc. Cambridge Philos. Soc. 1997. V. 122. P. 357-375.

[3] Фарбер М.Ш. Точность неравенств Новикова // Функц. анализ и его прилож. 1985. T. 19. № 1. C. 49-59.

[4] Новиков С. П. Гамильтонов формализм и многозначный аналог теории Морса // УМН. 1982. T. 37. C. $3-49$.

[5] Audin M. The Topology of Torus Actions on Symplectic Manifolds. Basel: Birkhäuser, 1991.

[6] David S. Metzler Wall-crossing formula for the signature of symplectic quotients // Preprint math.SG/9809030.

School of Mathematical Sciences, Tel-Aviv University, Israel

Поступила в редакцию

E-mail: farber@math.tau.ac.il 